\title{
Molecular Dynamics Calculation of Critical Point of Nickel
}

\author{
Changrui Cheng ${ }^{1}$ and Xianfan $\mathrm{Xu}^{1,2}$
}

Received: June 28, 2005

\begin{abstract}
The critical point of nickel and the phase diagram near the critical point are numerically evaluated using molecular dynamics (MD) computations. Thermodynamic states on the phase diagram are calculated for a homogeneous material at equilibrium states. Isothermal lines on $p-v$ diagrams are constructed at temperatures below and above the critical temperature, and the liquid-gas coexistence lines and regimes are obtained. The critical point of nickel is obtained as $T_{c}=9460 \pm 20 \mathrm{~K}, \rho_{c}=2560 \pm 100 \mathrm{~kg} \cdot \mathrm{m}^{-3}$, and $p_{c}=1.08 \pm$ $0.01 \mathrm{GPa}$. The method used in this work can be used to estimate thermodynamic properties of other materials at high temperature/pressure.
\end{abstract}

KEY WORDS: critical point; equation of state; molecular dynamics; phase diagram.

\section{INTRODUCTION}

The critical point is an essential thermodynamic property of a material that determines its characteristics and behavior in many ways [1]. The critical point is defined by the critical temperature $T_{c}$, the critical pressure $p_{c}$, and the critical density $\rho_{c}$. The critical temperature can be understood as the highest temperature at which liquid and vapor phases coexist in equilibrium. Knowledge of the critical point of a material will help to understand many phase-change processes, such as evaporation, boiling, and other fast transitions between liquid and gas at high temperature and high pressure.

\footnotetext{
${ }^{1}$ School of Mechanical Engineering, Purdue University, West Lafayette, Indiana 47907, U.S.A.

${ }^{2}$ To whom correspondence should be addressed. Email: xxu@ecn.purdue.edu
} 
Most work on the critical point was done on materials that are gases and liquids at atmospheric condition. On the other hand, it is also important to study the critical point of materials that are solid at room temperature, among which are many commonly - used metals. In some metal processing such as laser ablation, plasma processing, and ion deposition, the material could be heated to thermodynamic states close to or even above the critical point, and phase changes could occur in a very short period of time. For these processes, it is crucial to know the critical point and the phase diagram of metals to better understand how the material behaves during these processes and how to better control and optimize the processing parameters.

There are not many experimental data available for the critical point of metals, mainly because their critical temperatures and pressures are often very high and therefore are difficult to be measured accurately. In this work, we use the molecular dynamics (MD) technique, a first principles method, to estimate the critical point and the phase diagram near the critical point. Equilibrium thermodynamic states at various pressures, densities, and temperatures are obtained. Isothermal lines on a $p-v$ diagram are constructed, and the liquid-gas coexistence regimes are obtained, from which the critical point is determined. Nickel is used as an example in this work; however, the method can be extended to other materials if the potential functions determining interatomic interactions are known.

\section{NUMERICAL APPROACH}

In molecular dynamics (MD) simulations, interactions between molecules or atoms in a material are determined by a prescribed potential function. The motion of each molecule is governed by this potential and follows Newton's law. The positions and velocities of all molecules at each time step are tracked, and the macroscopic quantities (temperature, pressure, density, etc.) in the material are calculated from the statistical analysis of positions, velocities, and forces of the molecules at locations of interest. With very few assumptions of the material properties and the process, MD has particular advantages in dealing with complicated problems in materials, fluid dynamics, biology, and chemistry, and has been widely used for many different applications successfully [2-4].

The pair-wise Morse potential is commonly used for fcc (face centered cubic) metals, and is applied in this work to describe interactions among nickel atoms,

$$
\Phi\left(r_{i j}\right)=D\left[e^{-2 b\left(r_{\mathrm{ij}}-r_{\varepsilon}\right)}-e^{-b\left(r_{\mathrm{ij}}-r_{\varepsilon}\right)}\right]
$$


In Eq. (1), $D$ is the total dissociation energy, $r_{\varepsilon}$ is the equilibrium distance, and $b$ is a constant, with values of $0.4205 \mathrm{eV}, 0.278 \mathrm{~nm}$, and $14.199 \mathrm{~nm}^{-1}$, respectively [5]. The Morse potential is chosen because it has been shown to be a good approximation to the atomic interactions in fcc metals such as nickel, and is capable of predicting many material properties [5]. Other potential functions, such as the EAM (embedded atomic method), have been proposed [6,7]; however, it is not conclusive from the available literature whether it has advantages over the Morse potential. On the other hand, the Morse potential has a simpler form which allows us to evaluate a large number of thermodynamic points to obtain the phase diagram and the critical point.

The procedure of the MD computation is briefly described below. At each time step, the total force, velocity, and position of each atom are calculated. The total force acting on atom $i$ is simply the summary of force vectors from all neighboring atoms:

$$
\overrightarrow{F_{i}}=\sum_{j \neq i} \overrightarrow{F_{j i}}=\sum_{j \neq i} F\left(r_{j i}\right) \overrightarrow{r_{j i}^{0}}
$$

where $F\left(r_{j i}\right)$ is calculated from the Morse potential as

$$
F\left(r_{j i}\right)=-\frac{\partial \Phi\left(r_{i j}\right)}{\partial r}=2 D b\left[e^{-2 b\left(r_{i j}-r_{\varepsilon}\right)}-e^{-b\left(r_{i j}-r_{\varepsilon}\right)}\right]
$$

The interaction among atoms is neglected when $r$ is larger than the cut-off distance (taken as $2.46 r_{\varepsilon}$ in this work because the potential and force are negligible at this distance). After the total force for each atom is obtained, the velocity and position at the new time step are calculated using the modified Verlet algorithm [8], where the velocity and position of atom $i$ are calculated from

$$
\begin{gathered}
\overrightarrow{r_{i}}(t+\delta t)=\overrightarrow{r_{i}}(t)+\overrightarrow{v_{i}}\left(t+\frac{\delta t}{2}\right) \delta t \\
\overrightarrow{v_{i}}\left(t+\frac{3}{2} \delta t\right)=\overrightarrow{v_{i}}\left(t+\frac{\delta t}{2}\right)+\frac{\overrightarrow{F_{i}}(t+\delta t)}{m} \delta t
\end{gathered}
$$

The target material in this calculation is composed of 403,200 atoms and has dimensions of $10.62 \times 10.62 \times 39.66 \mathrm{~nm}^{3}$ at $300 \mathrm{~K}$. Periodical boundary conditions are applied on the three directions. We used a larger size in one direction to examine the homogeneity of the computed parameters such as temperature and pressure. 
Calculations of temperature and pressure are described as follows. The temperature $T$ is calculated by summing the kinetic energy of all the atoms:

$$
T=\frac{1}{3 N k_{\mathrm{B}}} m \sum_{i=1}^{N} \sum_{k=1}^{3} v_{i, k}^{2}
$$

where $N$ is the total number of atoms, $k_{B}$ is the Boltzmann constant, and $m$ is the mass of the atom. $k$ represents the spatial coordinate, and $v_{i, k}$ is the velocity of atom; $i$ at the $k$-th coordinate obtained from the MD calculations.

The pressure of the material $p$ is evaluated from the virial theory [9];

$$
p=\rho k_{\mathrm{B}} T+\frac{1}{3 V}\left\langle\sum_{i=1}^{N} \sum_{j<i} \overrightarrow{F_{i j}} \cdot \overrightarrow{r_{i j}}\right\rangle
$$

where $\rho$ is the number density and $V$ is the total volume. The first part $\left(\rho k_{\mathrm{B}} T\right)$ arises from the random motion of atoms without considering forces among atoms, similar to the pressure for ideal gases, $p=R T / v$. The second part $\left(\frac{1}{3 V}\left\langle\sum_{i=1}^{N} \sum_{j<i} \overrightarrow{F_{i j}} \cdot \overrightarrow{r_{i j}}\right\rangle\right)$ takes into account the interacting forces among atoms, where the attractive internal force generates a negative pressure and the repulsive force adds a positive pressure.

Since the simulated material is in equilibrium, the pressure, temperature, and specific volume are average values of the whole domain. For the states when the material is a mixture of liquid and gas, the specific volume will be the spatially averaged value which is the total volume divided by the total mass.

The isothermal lines on a $p-v$ diagram near the critical point are calculated. For each temperature, 10-20 points are calculated to form an isothermal line on the $p-v$ diagram. Each point is equilibrated for at least $50 \mathrm{ps}$, and the calculation continues until the pressure does not change in another $30 \mathrm{ps}$ to ensure an equilibrium state. After one point on the $p-v$ diagram is obtained, the same procedure is repeated for the next point with a different specific volume. Since changing the specific volume will change the temperature, velocity-scaling [10] is used so that the material's temperature reaches the expected value under the new equilibrium condition.

The calculated pressure/specific volume pairs at a constant temperature will constitute an isothermal line on the $p-v$ diagram. The procedures are then repeated to obtain isothermal lines at different temperatures. The locations on the isothermal lines where the slopes change form the 
liquid-gas saturation lines (binodes), as will be seen in the next section. The peak of the two saturation lines is the critical point, and its parameters of temperature, pressure, and density can then be determined.

\section{RESULTS AND DISCUSSION}

To evaluate the MD method, the specific heat of nickel at constant pressure is calculated. First, an equilibrium state of a nickel target at $300 \mathrm{~K}$ and $1 \mathrm{~atm}$ is obtained. Then, a certain amount of energy $Q$ is added into the material, and the material reaches another equilibrium state with a temperature increase $\Delta T$. The value of $c_{p}$ is then obtained from

$$
c_{p}=\frac{Q}{M \Delta T}
$$

where $M$ is the mass of the material. The calculated specific heat at $330 \mathrm{~K}$ and $1 \mathrm{~atm}$ is $469 \pm 8 \mathrm{~J} \cdot \mathrm{kg}^{-1} \cdot \mathrm{K}^{-1}$ for nickel, less than $3 \%$ higher than the literature value of $456 \mathrm{~J} \cdot \mathrm{kg}^{-1} \cdot \mathrm{K}^{-1}$.

We now discuss the calculated isotherms on a $p-v$ diagram. Figure 1 shows an isothermal line at $9300 \mathrm{~K}$. To illustrate that the line is indeed isothermal, the temperature at each point is also plotted. It is seen that the temperature variation is within the range of $9300 \pm 3 \mathrm{~K}$. A liquidgas coexistence region can be identified in Fig. 1 where the pressure stays constant. At the left side of the isotherm, the pressure decreases rapidly with an increase of specific volume until it reaches $0.2898 \times 10^{-3} \mathrm{~m}^{3} \cdot \mathrm{kg}^{-1}$ (corresponding to a density of $3450 \mathrm{~kg} \cdot \mathrm{m}^{-3}$ ) and a pressure of $0.99 \mathrm{GPa}$,

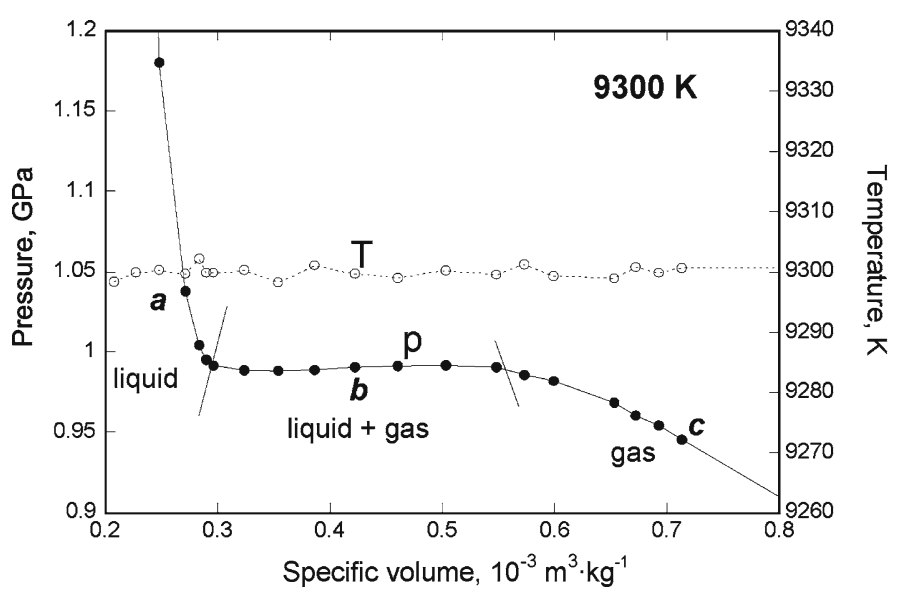

Fig. 1. Isotherm at $9300 \mathrm{~K}$. 
i.e., the point $p=0.99 \mathrm{GPa}, v=0.2898 \times 10^{-3} \mathrm{~m}^{3} \cdot \mathrm{kg}^{-1}$, and $T=9300 \mathrm{~K}$ is a point on the liquid-gas saturation line. As the specific volume increases further, the pressure remains constant. (A close examination of this plateau reveals a very small van der Waals-like loop, which is due to the computational size used. The van der Waals-like loop is more obvious when a smaller calculation domain is used.) The pressure starts to decrease again with an increase of the specific volume from $v=0.5609 \times 10^{-3} \mathrm{~m}^{3} \cdot \mathrm{kg}^{-1}$ (corresponding to a density of $1783 \mathrm{~kg} \cdot \mathrm{m}^{-3}$ ). This indicates that the point $p=0.99 \mathrm{GPa}, v=0.5609 \times 10^{-3} \mathrm{~m}^{3} \cdot \mathrm{kg}^{-1}$ is the other point on the liquidgas saturation line at $T=9300 \mathrm{~K}$. Between these two points, the material is a mixture of liquid and gas states, although the mass fraction of each phase changes with the specific volume.

The phases of the material along the isotherm are confirmed by the observation of the atomic structures. In Fig. 2, the material structures corresponding to three states in Fig. 1 are shown. Each black dot in Fig. 2 represents one atom. The more uniform liquid and gas structures can be seen from the atomic distribution in Figs. 2a (liquid) and c (gas), while at state $b$, which is a mixture of liquid and gas, the material structure is not as uniform. The difference in uniformity at these three states is also shown in Fig. 3, the snapshot of atomic number density at different locations along the long axis of the calculation domain $(39.66 \mathrm{~nm}$ at room temperature). It is seen that the fluctuation of density at state $b$ is larger than those at the other two states, indicating the coexistence of gas and liquid state in state $b$ while the other two states have more uniform density throughout the calculation domain. However, it is seen from Fig. 2c that the atomic distribution is not completely uniform, indicating that a more complex structure may exist in the gaseous phase such as dimers and higher-order clusters, particularly near the binodal line. This indicates that
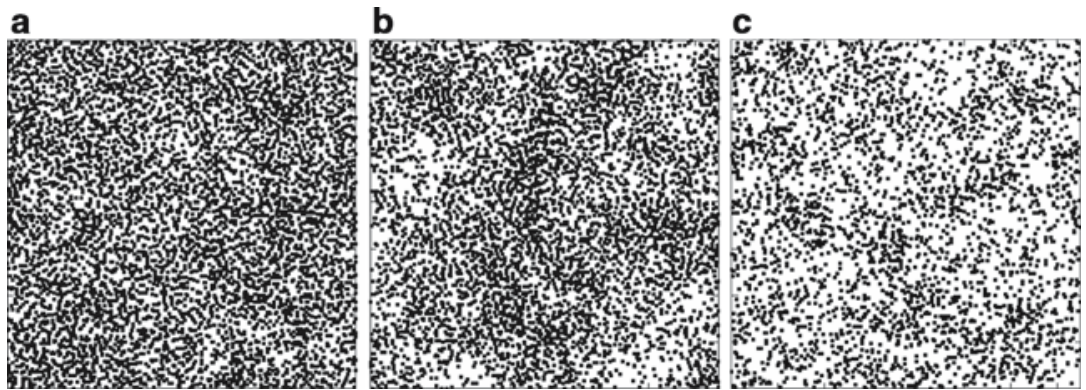

Fig. 2. Atomic structure of (a) liquid, (b) liquid-gas coexistence, and (c) gas phases as marked on the phase diagram in Fig. 1. 


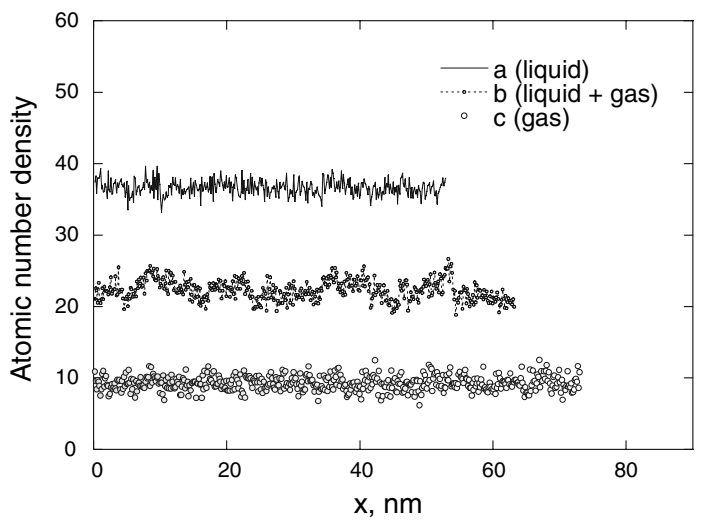

Fig. 3. Atomic number density at several locations on the phase diagram in Fig. 1 ( $a$, liquid; $b$, liquid-gas coexistence; $c$, gas).

atomic distribution plots may not be a reliable way to determine the phase of a material. Another way to distinguish the phases is to compute the radial distribution functions at the locations of interest. The radial distribution function describes the distribution of the distances between any two atoms in the material, and it represents the relative probability to find a pair of atoms separated by distance $r$. The definition of the radial distribution function $g(r)$ is given as

$$
g(r)=\left\langle\frac{n(r+\Delta r)-n(r)}{4 \pi r^{2} \Delta r} \frac{V}{N}\right\rangle
$$

where $n(r)$ is the number of atoms within a distance $r$ from a certain atom, $\Delta r$ is the distance step (chosen as $0.01 \mathrm{~nm}$ in this calculation to achieve an appropriate resolution of $g(r)), V$ is the total volume, and $N$ is the total atom number. The radial distribution function is always calculated and averaged for a large number of atoms.

Typical radial distribution functions for solid, liquid, and gas of nickel are shown in Fig. 4. Crystal solids have regular lattice structure, so distances between atoms have a regular distribution which is represented by oscillations of $g(r)$ in both long and short ranges. For liquids, the short-range order remains, but the long-range order disappears. Both the short-range and long-range orders vanish for gases. Therefore, the radial distribution, function is commonly used to illustrate the structure and the phase of a material [8]. The radial distribution functions at states $a, b$, and $c$ in Fig. 1 are calculated and shown in Fig. 5. Note that the curves of states $b$ and $c$ are raised for clarity. For state $a$, the radial distribution 


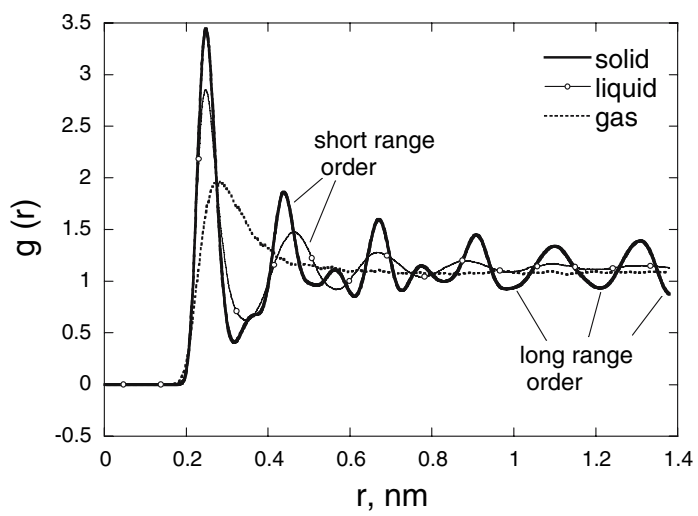

Fig. 4. Radial distribution function for solid, liquid, and gas.

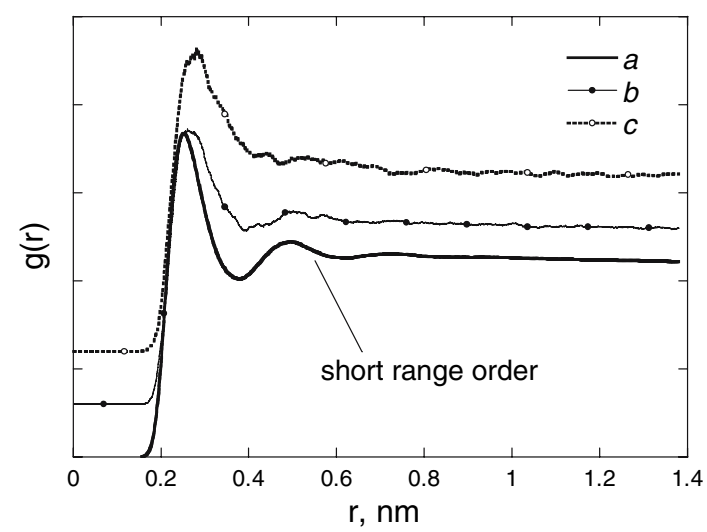

Fig. 5. Radial distribution function $g(r)$ at several locations on the phase diagram in Fig. 1 ( $a$, liquid; $b$, liquidgas coexistence; $c$, gas). Curves of $b$ and $c$ are raised for clarity.

function shows short-range order as that of a liquid. (The radial distribution function does not return to 1 at $1.4 \mathrm{~nm}$, which is likely due to the truncation error in the calculation.) For the radial distribution function at state $c$, the short-range order disappears completely, which indicates that state $c$ is in the gas phase. The profile of $g(r)$ for state $b$ is between those of $a$ and $c$, and the short-range order is only observed partially.

Isotherms are also constructed for several other temperatures as shown in Fig. 6. It is seen that at temperatures lower than $9450 \mathrm{~K}$, 


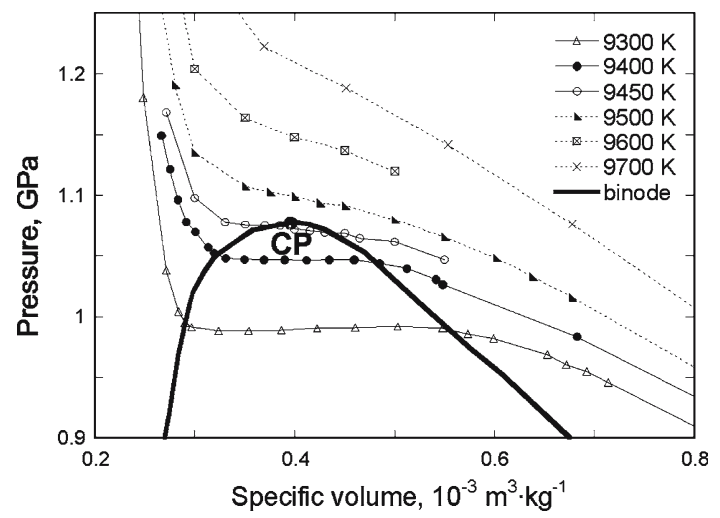

Fig. 6. Calculated isotherms on $p-v$ diagram around the critical point.

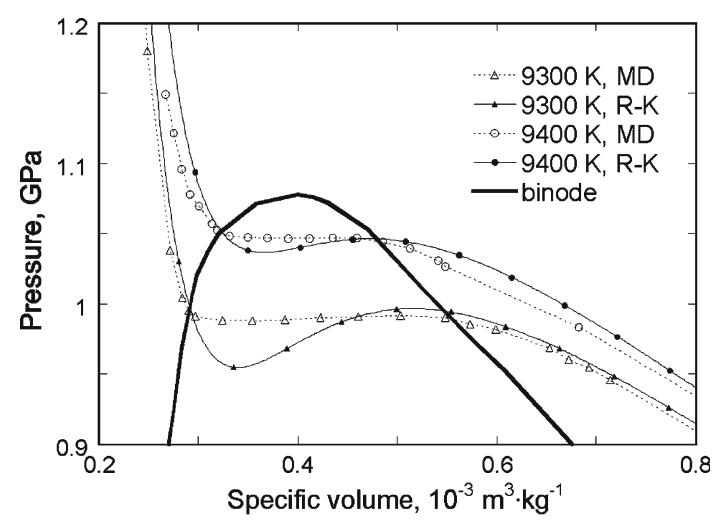

Fig. 7. Isotherms calculated from MD simulations and from the Redlich-Kwong equation.

there is always a liquid-gas coexistence region where the pressure does not change with the specific volume. The boundaries of the liquid-gas co-existence regions form the binode lines. When the temperature is higher than $9500 \mathrm{~K}$, the pressure decreases monotonically with an increase of the specific volume and the plateau of pressure with respect to specific volume does not exist. Therefore, the critical temperature lies between 9450 and $9500 \mathrm{~K}$.

Further calculations at temperatures between 9450 and $9500 \mathrm{~K}$ found that the parameters of the critical point are: $T_{\mathrm{c}}=9460 \pm 20 \mathrm{~K}, \rho_{\mathrm{c}}=2560 \pm$ $100 \mathrm{~kg} \cdot \mathrm{m}^{-3}$, and $p_{\mathrm{c}}=1.08 \pm 0.01 \mathrm{GPa}$. There are only a few values in the 
literature for the critical point of nickel: $9576 \mathrm{~K} / 2293 \mathrm{~kg} \cdot \mathrm{m}^{-3} / 1.12 \mathrm{GPa}$ [11], $7810 \mathrm{~K} / 2210 \mathrm{~kg} \cdot \mathrm{m}^{-3} / 0.49 \mathrm{GPa}$ [12], and $9100 \pm 150 \mathrm{~K} / 0.9 \pm 0.1$ $\mathrm{GPa}$ [13]. These values are either extrapolated from low-temperature data using semi-empirical equations of state, or from shock-compression experiments.

Another outcome of the MD results is the equation of state (EOS), which is often unavailable at high temperature and high pressure. In this calculation, we tried to obtain simple two-constant forms of an equation of state, such as van der Waals, Redlich-Kwong, and Berthelot equations and found that the Redlich-Kwong equation best matches the MD data. The Redlich-Kwong equation has the form,

$$
p=\frac{n R T}{v-b}-\frac{a}{v(v+b) \sqrt{T}}
$$

where $a$ and $b$ are constants. These constants can be obtained from the requirements that both the first and second derivatives of the isotherm are zero at the critical point, which results in

$$
a=0.42748 \frac{(n R)^{2} T_{\mathrm{c}}^{5 / 2}}{p_{\mathrm{c}}}, \quad b=0.08664 \frac{n R T_{\mathrm{c}}}{p_{\mathrm{c}}}
$$

Using the critical point parameters obtained from $\mathrm{MD}, a$ and $b$ are found to be

$$
a=69113 \mathrm{~m}^{6} \cdot \mathrm{kg}^{-2} \cdot \mathrm{K}^{1 / 2}, \quad b=1.0749 \times 10^{-4} \mathrm{~m}^{3} \cdot \mathrm{kg}^{-1}
$$

Using these values, Eq. (9) is plotted together with isotherms obtained from MD calculations. For the isotherms calculated from Eq. (9), the sections between the binodes should be ignored. As mentioned earlier, the Redlich-Kwong equation matches the MD results the best, while other forms of the equation of state do not match MD results as well (not shown in the figure).

\section{CONCLUSION}

In this work, the phase diagram of nickel near the critical point is calculated using molecular dynamics simulations. Isotherms near the critical temperature are constructed on a $p-v$ diagram. Regions where liquid and gas co-exist and the binode lines are obtained. The critical point obtained in this work is in good agreement with literature values. It is also found that the Redlich-Kwong equation can best represent the phase diagram near the critical point compared with other forms of EOS. 


\section{ACKNOWLEDGMENT}

Support to this work by the National Science Foundation is gratefully acknowledged.

\section{REFERENCES}

1. M. J. Moran and H. N. Shapiro, Fundamentals of Engineering Thermodynamics (John Wiley \& Sons, New York, 1996), pp. 72-74, 491.

2. Y. Guissani and B. Guillot, J. Chem. Phys. 104:7633 (1996).

3. D. O. Dunikov, S. P. Malyshenko, and V. V. Zhakhovskii, J. Chem. Phys. 115: 6623 (2001).

4. A. Shibinsky, S. V. Buldyrev, G. Franzese, G. Malescio, and H. E. Stanley, Phys. Rev. E 69:061206 (2004).

5. L. A. Girifalco and V. G. Weizer, Phys. Rev. 114:687 (1959).

6. S. M. Foiles, M. I. Baskes, and M. S. Daw, Phys. Rev. B 33:7983 (1986).

7. M. S. Daw, S. M. Foiles, and M. I. Baskes, Mater. Sci. Rep. 9:251 (1993).

8. M. P. Allen and D. J. Tildesley, Computer Simulation of Liquids (Clarendon Press, Oxford, 1987), pp. 78-80.

9. J. M. Haile, Molecular Dynamics Simulation: Elementary Methods (John Wiley \& Sons, New York, 1992), p. A1.

10. H. J. C. Berendsen, J. P. M. Postma, W. F. van Gunsteren, A. DiNola, and J. R. Haak, J. Chem. Phys. 81:3684 (1984).

11. D. A. Young and B. J. Alder, Phys. Rev. A 3:364 (1971).

12. M. M. Martynyuk, Russ. J. Phys. Chem. 57:810 (1983).

13. D. N. Nikolaev, V. Y. Ternovoi, A. A. Pyalling, and A. S. Filimonov, Int. J. Thermophys. 23:1311 (2002). 